\title{
Влияние энергии бомбардирующих электронов на проводимость эпитаксиальных слоев $n-4 H$-SiC (CVD)
}

\author{
(C) В.В. Козловский ${ }^{1}$, А.А. Лебедев ${ }^{2}$, А.М. Стрельчук ${ }^{2}$, К.С. Давыдовская ${ }^{2}$, \\ А.Э. Васильев ${ }^{1}$, Л.Ф. Макаренко ${ }^{3}$ \\ ${ }^{1}$ Санкт-Петербургский политехнический университет Петра Великого, \\ 195251 Санкт-Петербург, Россия \\ ${ }^{2}$ Физико-технический институт им. А.Ф. Иоффе Российской академии наук, \\ 194021 Санкт-Петербург, Россия \\ ${ }^{3}$ Белорусский государственный университет, \\ 220030 Минск, Республика Беларусь \\ E-mail: kozlovski@physics.spbstu.ru
}

(Получена 7 сентября 2016 г. Принята к печати 14 сентября 2016 г.)

\begin{abstract}
Проведено исследование электрофизических характеристик эпитаксиальных слоев $n$ - $4 H$-SiC (CVD) при его облучении электронами с энергиями 0.9 и $3.5 \mathrm{MэB.} \mathrm{Показано,} \mathrm{что} \mathrm{скорость} \mathrm{удаления} \mathrm{доноров} \mathrm{увеличивается}$ почти в 4 раза с ростом энергии бомбардирующих электронов в 4 раза, хотя сечение образования первичных радиационных дефектов (пар Френкеля в подрешетке углерода), ответственных за компенсацию проводимости материала, в этом диапазоне практически не зависит от энергии. Предположено, что причиной наблюдаемых различий является влияние первично выбитых атомов. Во-первых, с ростом энергии первично выбитых атомов начинают сказываться каскадные процессы. Во-вторых, увеличивается среднее расстояние между генетически родственными парами Френкеля и как следствие увеличивается доля не рекомбинирующих при облучении дефектов. Проведены оценки радиуса рекомбинации пары Френкеля в подрешетке углерода и возможного зарядового состояния рекомбинирующих компонент.
\end{abstract}

DOI: 10.21883/FTP.2017.03.44199.8399

\section{1. Введение}

Облучение полупроводниковых приборов пучками электронов с энергией порядка несколько МэВ находит широкое применение для регулирования ряда параметров полупроводниковых приборов: для кремниевых силовых импульсных приборов - это прежде всего частотные характеристики, связанные со временем жизни неравновесных носителей заряда [1]; для арсенидгаллиевых диодов - это обратные рабочие напряжения, ограничиваемые токами утечки по боковой поверхностной фаске приборов [2,3]. Физической основой такого применения электронного облучения является образование на глубинах полупроводника до сотен микрон радиационных дефектов (РД), являющихся эффективными центрами рекомбинации (для $\mathrm{Si}$ ) и компенсации (для $\mathrm{GaAs}$ ). Радиационное дефектообразование в базовых материалах современной полупроводниковой электроники ( $\mathrm{Si}, \mathrm{GaAs})$ исследуется на протяжении последних пятидесяти лет, результаты которого изложены в обзорах и монографиях [4-6].

В последние годы в полупроводниковой электронике (в том числе силовой и высокочастотной) стремительно развивается применение нового широкозонного материала - карбида кремния. В 70-х годах XX столетия начались работы по исследованию радиационных дефектов в этом материале [7]. Было установлено, что облучение, как правило, ведет к компенсации проводимости материала $n$-типа проводимости [8-10]. Успехи в создании высококачественных слоев эпитаксиального политипа
n-4H-SiC методом CVD, достигнутые за последние двадцать лет, позволили приступить не только к выпуску промышленных приборов (например, диодов Шоттки) на его основе, но и по-новому систематически подойти к исследованию РД в карбиде кремния. В частности, в работах [11-13] с помощью низкоэнергетического (до 200 кэВ) электронного облучения было установлено, что основные РД, обусловливающие компенсацию проводимости $n-\mathrm{SiC}$ (CVD) (так называемые Z1/Z2 и $E H 6 / 7$ дефекты), связаны с компонентами пары Френкеля ПФ (вакансия и межузельный атом) в одной из подрешеток $\mathrm{SiC}$, а именно в углеродной. Нами в предыдущей работе [14] было установлено, что уменьшение концентрации нескомпенсированных доноров в $n$-SiC (CVD) при облучении электронами с энергией 0.9 МэВ обусловлено именно компенсацией проводимости материала собственными радиационными дефектами, а не пассивацией легирующей примеси путем образования комплексов (дефект+атом мелкой легирующей примеси).

В вышеупомянутых работах эксперименты проводились при энергиях бомбардирующих электронов достаточных для образования только одиночных близко расположенных (иногда называемых „генетически родственных“) пар вакансия - межузельный атом (пара Френкеля (ПФ)). В радиационной физике твердого тела такой вид облучения часто называют модельным. С ростом энергии бомбардирующих электронов первично выбитый атом (ПВА) способен сам выбивать атом решетки: увеличивается среднее число смещенных атомов $v$, приходящихся на каждый ПВА (величина $v$ чаще 
называется коэффициентом размножения) и происходит образование каскада смещений. Цель настоящей работы - сравнение процессов компенсации проводимости $n$-SiC при модельном облучении $\left(E_{e}=0.9 \mathrm{MэB}\right)$ и при „надпороговом“ (по отношению к образованию каскада) облучении с коэффициентом размножения порядка два $\left(E_{e}=3.5 \mathrm{MэB}\right)$. Практическая цель работы состояла в том, чтобы получить с помощью электронного облучения высокоомные слои $n-\mathrm{SiC}$ толщиной в сотни микрон и объяснить зависимости эффекта компенсации проводимости от дозы облучения.

\section{2. Экспериментальные детали}

В настоящей работе были исследованы диоды Шоттки на основе эпитаксиальных 4H-SiC (CVD)-структур производства фирмы CREE (США) [15]. Схема облучения диода представлена на рис. 1. Размеры образцов составляли $1.07 \times 0.66 \times 0.37$ мм. Эпитаксиальная структура состоит из $n^{+}$-подложки и $n$-слоя (CVD) с концентрацией нескомпенсированной донорной примеси $\left(N_{d}-N_{a}\right) \sim(6-7) \cdot 10^{15} \mathrm{~cm}^{-3}$. Омический $\mathrm{Ni} / \mathrm{Ag}$-контакт (катод) был сформирован на обратной стороне подложки. Барьерный контакт (Al) представлял собой полоску шириной 70 мкм и толщиной 4мкм, созданную по периметру лицевой стороны образца. Обучение электронами с энергией 0.9 МэВ проводилось на ускорителе РТЭ-1В; с энергией 3.5 МэВ - на ускорителе ЭЛУ-4. Можно считать, что при электронном облучении дефекты вводились равномерно по объему образцов, поскольку толщина облучаемых образцов $\mathrm{SiC}$ была значительно меньше длины пробега электронов. Максимальная доза облучения составляла $\sim 1.5 \cdot 10^{17} \mathrm{~cm}^{-2}$. Концентрации нескомпенсированной донорной примеси $\left(N_{d}-N_{a}\right)$ в исходных и облученных образцах определялись из вольт-фарадных характеристик ВФХ $(C-U)$ на стандартной установке с параллельной схемой замещения и синусоидальной частотой 10 кГц при комнатной температуре. Дифференциальное последовательное сопротивление (и проводимость) в исходных и облученных диодах определялось из вольт-амперных характеристик $(I-V)$ при максимальных измеряемых токах (10 мА) или напряжениях (10 B).

Скорость изменения концентрации рассчитывалась по формуле

$$
\eta_{e}=\left(N_{0}-N\right) / D
$$

где $N_{0}$ и $N-$ концентрации $\left(N_{d}-N_{a}\right)$ в эпитаксиальном слое до и после облучения; $D$ - доза облучения.

\section{3. Экспериментальные результаты}

На рис. 2 представлены зависимости концентрации $\left(N_{d}-N_{a}\right)$ от дозы облучения при двух энергиях электронов. Экспериментальные точки, полученные для обеих энергий, весьма неплохо описываются прямыми

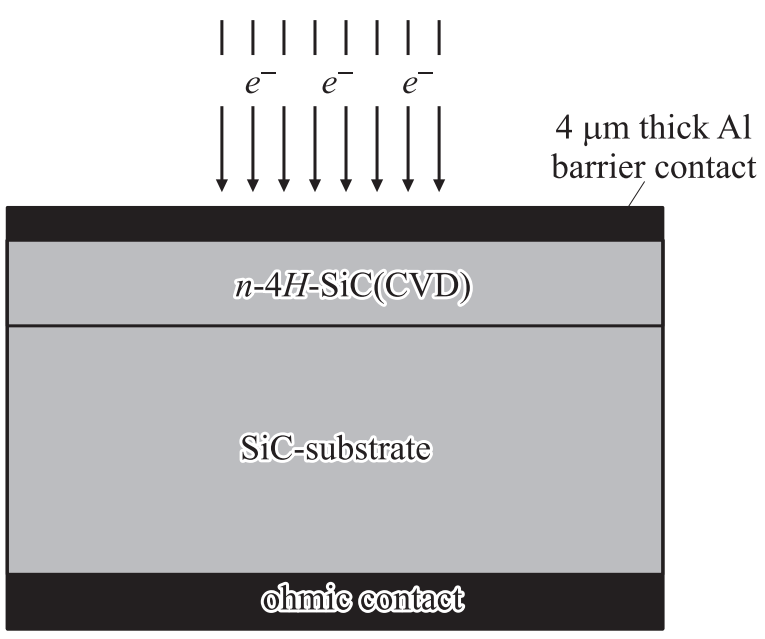

Рис. 1. Схематическое изображение процесса облучения исследованных диодов Шоттки на $\mathrm{SiC}$.

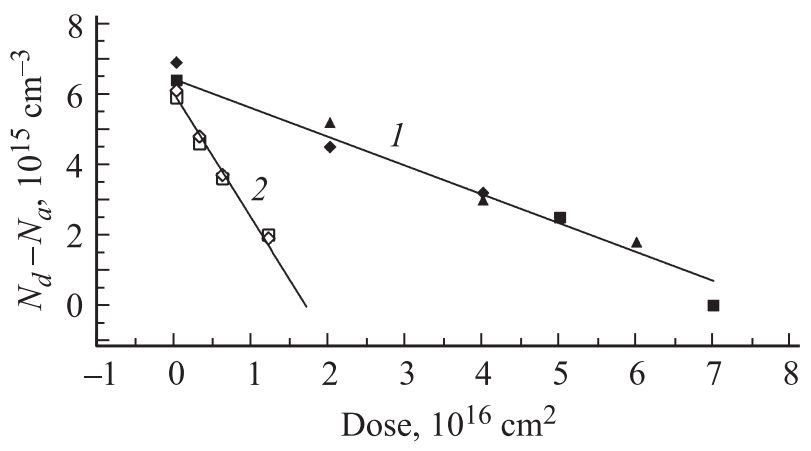

Рис. 2. Зависимость $N_{d}-N_{a}$ от дозы облучения для случая облучения электронами с энергией 0.9 (1) и $3.5 \mathrm{MэB} \mathrm{(2).}$ Разные символы соответствуют разным диодам.

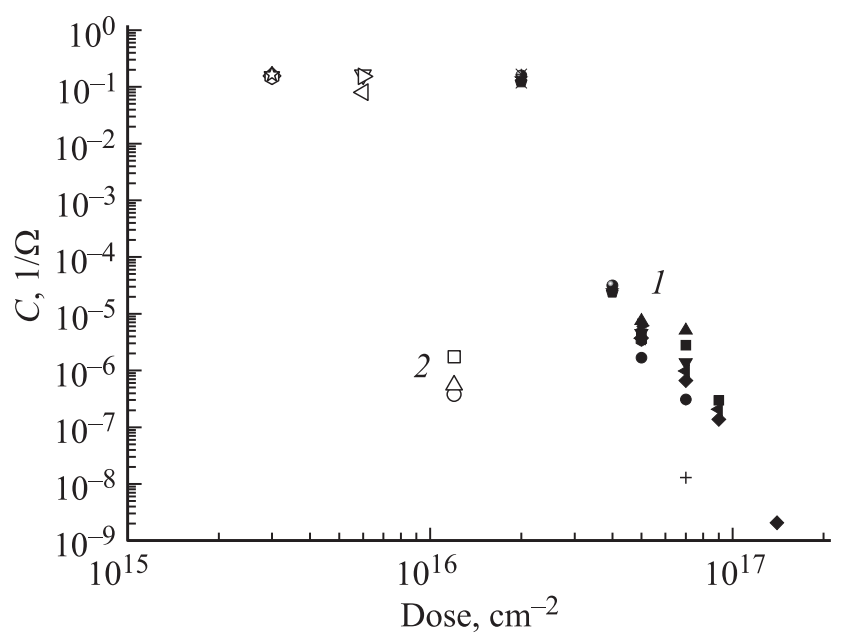

Рис. 3. Зависимость проводимости в прямом направлении диодов Шоттки от дозы облучения для случая облучения электронами с энергией 0.9 (1) и 3.5 МэВ (2). Разные символы соответствуют разным диодам. 
линиями (прямая 1 и 2). Расчет скорости изменения концентрации по формуле (1) для энергии 0.9 МэВ (прямая 1 ) позволил получить значение $\eta_{e} \sim 0.1 \mathrm{~cm}^{-1}$. Значение $\eta_{e}$, определенное для энергии электронов $3.5 \mathrm{MэB}$, составляет $\sim 0.4 \mathrm{~cm}^{-1}$. Такое большое отличие (почти в 4 раза) в значениях $\eta_{e}$ несколько удивительно, так как сечение образования радиационных дефектов в диапазоне 1-5 МэВ практически не зависит от энергии электронов [4-6].

На рис. 3 представлены зависимости проводимости $n-4 H-\mathrm{SiC}$ от дозы облучения для двух энергий электронов. Обе зависимости имеют одинаковый вид и характеризуются резким (на 5 порядков) уменьшением проводимости с ростом дозы облучения. Критические дозы облучения, при которых наблюдается резкий скачок, составляют $\sim(2.0-2.5) \cdot 10^{16} \mathrm{~cm}^{-2}$ для электронов с энергией $0.9 \mathrm{MэB} \mathrm{и}(5-6) \cdot 10^{15} \mathrm{~cm}^{-2}$ для электронов с энергией $3.5 \mathrm{MэB}$. Измерения $\mathrm{BAX}$, так же как и ВФХ, свидетельствуют о том, что эффективность воздействия увеличивается почти в 4 раза с ростом энергии электронов. При этом, что сечение образования РД не изменяется в этом диапазоне энергий.

\section{4. Анализ результатов и сравнение с расчетами}

Для объяснения экспериментальных результатов рассмотрим процесс образования РД в карбиде кремния, особое внимание уделив дефектам в подрешетке углерода, которые, как указано в разд. 1, формируют компенсирующие центры в $n$-SiC при электронном облучении. Атому решетки с массой $M$ (массовым числом $A$ ) при упругом взаимодействии с релятивистским электроном с энергией $E_{e}$ и массой $m$ может быть передана энергия $E$, величина которой лежит в пределах от нуля до максимальной:

$$
\begin{aligned}
E_{\max } & =2 E_{e}\left(E_{e}+2 m_{e} c^{2}\right) / M c^{2} \\
& =E_{e}\left(E_{e}+1.022\right) /(469 \cdot A)(\mathrm{M \ni B}) .
\end{aligned}
$$

При $E_{\max }$, равной пороговой энергии образования дефекта $E_{d}$, формула (2) определяет граничную энергию электрона $E_{\text {bound, }}$, выше которой начинают генерироваться первичные точечные дефекты вакансия-межузельный атом (пара Френкеля ПФ). В работах [11,12] было показано, что при облучении карбида кремния минимальные граничные энергии электронов, при которых наблюдается образование РД, в подрешетке кремния составляют $\sim 250$ кэВ, а в подрешетке углерода $\sim 90$ кэВ. Пороговые энергии образования РД в карбиде кремния, определенные по формуле (2), составляют для подрешетки кремния $E_{d} \sim 24$ эВ, для подрешетки углерода $E_{d} \sim 18$ эВ. Рассеяние, имеющее место при столкновениях, приводящих к смещениям, главным образом связано с кулоновским взаимодействием электрона с ядром мишени. Релятивистское кулоновское рассеяние электронов показывает, что сечение смещений по мере увеличения энергии бомбардирующих электронов постепенно возрастает от нуля при некой граничной энергии электронов (при облучении карбида кремния граничные энергии равны 90 и 250 кэВ) и затем становится постоянным. Поскольку выбранные нами в настоящей работе энергии 0.9 и $3.5 \mathrm{MэB} \mathrm{значительно} \mathrm{превосходят} E_{\text {bound }}$, то сечение рассеяния можно оценивать по упрощенной формуле Мак-Кинли-Фешбаха [16]:

$$
\begin{aligned}
\sigma_{d} & =\left(1 / 4 \pi \varepsilon_{0}\right)^{2}\left(2 \pi Z^{2} e^{4} / E_{d} M c^{2}\right) \\
& =\left[140 Z^{2} / A E_{d}(\text { эВ) }] \text { (барн }\right) .
\end{aligned}
$$

$\mathrm{B}$ настоящей работе, как указано выше, для $E_{d}$ были приняты значения 24 (Si) и 18 (С) эВ. При таких пороговых энергиях сечения образования РД по формуле (3) составляют $\sim 40$ барн $(\mathrm{Si})$ и 23 барн $(\mathrm{C})$ и практически не зависят от энергии бомбардирующих электронов в диапазоне 1-10 МэВ.

Скорость генерации ПВА $\eta_{\text {FP }}$ за счет взаимодействия с налетающим электроном вычисляется как произведение сечения $\sigma_{d}$ на концентрацию атомов мишени $N_{\mathrm{Si}}$ или $N_{\mathrm{C}}$. Соответственно скорость генерации ПВА для бинарного полупроводника $\mathrm{SiC}$ была получена как сумма парциальных значений для атомов кремния и углерода. Тогда при значении концентрации регулярных атомов $N=4.8 \cdot 10^{22} \mathrm{~cm}^{-3}$ скорость генерации ПВА будет составлять $\sim 2 \mathrm{~cm}^{-1}$ для подрешетки кремния, а для подрешетки углерода $\sim 1 \mathrm{~cm}^{-1}$. Суммарная скорость генерации ПВА в подрешетках $\operatorname{SiC}\left(3 \mathrm{~cm}^{-1}\right)$ практически одинакова в диапазоне от 1 до $10 \mathrm{MэВ.} \mathrm{Таким}$ образом, генерация первичных радиационных дефектов за счет взаимодействия с налетающим электроном при энергиях 1 и 10 МэВ примерно одинакова и не может объяснить наблюдаемую разницу в скоростях удаления носителей заряда $\eta_{e}$. Причину таких различий следует искать в генерации дефектов уже не бомбардирующими электронами, а атомами отдачи; а также во вторичном радиационном образовании.

Поведение образовавшихся путем облучения полупроводника компонентов пары Френкеля (вакансия и межузельный атом) зависит от многих факторов, главными из которых являются температура облучения, примесный состав и уровень легирования конкретного полупроводника; энергия первично выбитого атома отдачи. В данной работе изменялся только один фактор - энергетический спектр ПВА. Как известно, энергетический спектр ПВА или количество атомов, первично выбитых релятивистским электроном из своих равновесных положений, как и в случае бомбардировки атомными частицами, распределяется приблизительно по закону обратного квадрата энергии [17]. Оценим среднюю энергию $\left\langle E_{\text {РКА }}\right\rangle$, которую получают ПВА на примере атома углерода при столкновении с релятивистским электроном. С учетом обратноквадратичной зависимости для вычисления средней энергии, которую 
Зависимость основных энергетических параметров атомов отдачи углерода и темпа генерации пар Френкеля в подрешетке углерода от энергии бомбардирующих электронов $E_{e}$

\begin{tabular}{c|r|r|c}
\hline \multirow{2}{*}{$\begin{array}{c}E_{e}, \\
\mathrm{M} \mathrm{B}\end{array}$} & $E_{\max }$ & $\left\langle E_{\mathrm{PKA}}\right\rangle$ & \multirow{2}{*}{$\begin{array}{c}\eta_{\mathrm{FP}} \\
\mathrm{cm}^{-1}\end{array}$} \\
\cline { 2 - 3 } & \multicolumn{2}{|c|}{$\ni \mathrm{B}$} & 1.2 \\
0.9 & 307 & 54 & 1.3 \\
1.0 & 359 & 57 & 1.5 \\
1.5 & 672 & 67 & 1.6 \\
2.0 & 1074 & 73 & 1.8 \\
2.5 & 1565 & 81 & 1.9 \\
3.0 & 2144 & 87 & 2.0 \\
3.5 & 2812 & 91 & 2.1 \\
4.0 & 3569 & 96 & 2.2 \\
4.5 & 4415 & 99 & 2.3 \\
5.0 & 5350 & 103 &
\end{tabular}

получают атомы полупроводника при столкновении с релятивистским электроном, применима формула, полученная для упругого резерфордовского рассеяния [17]:

$$
\left\langle E_{\mathrm{PKA}}\right\rangle=\left[E_{d} E_{\max } /\left(E_{\max }-E_{d}\right)\right] \ln \left(E_{\max } / E_{d}\right) .
$$

В таблице приведены значения средней и максимальной энергии атомов отдачи углерода в зависимости от энергии бомбардирующих электронов, рассчитанные по формулам (2) и (4). Как видно из таблицы, при облучении электронами с энергией $0.9 \mathrm{MэB}\left\langle E_{\mathrm{PKA}}\right\rangle$ углерода составляет $\sim 54$ эВ. При таком облучении в основном образуются одиночные близко расположенные („генетически родственные“ ) пары вакансия-межузельный атом (пара Френкеля (ПФ)). С ростом энергии бомбардирующих электронов первично выбитый атом способен сам выбивать атом решетки: увеличивается среднее число смещенных атомов $v$, приходящихся на каждый ПВА. Коэффициенты размножения $v$ оцениваются по формуле Кинчина-Пиза [18], модифицированной Зиглером [19]:

$$
v=\left\langle E_{\text {PKA }}\right\rangle / 2.5 E_{d} .
$$

Из формул (3) и (4) видно, что с ростом энергии электронов сечение образования дефектов не меняется, однако растет $\left\langle E_{\text {РКА }}\right\rangle$ и, следовательно, v. Так, для электронов с энергией $3.5 \mathrm{MэB},\left\langle E_{\text {РКА }}\right\rangle=91$ эВ и $v \sim 2$. Половина из общего количества ПВА углерода образуется при взаимодействии бомбардирующего электрона с атомами подрешетки, вторая половина ПВА образуется в каскадных процессах. Равенство двух „мощностей“ источников ПВА при $E_{e}=3.5$ МэВ было определяющим фактором в выборе такой энергии бомбардирующих электронов. Темп генерации ПФ $\left(\eta_{\mathrm{FP}}\right)$ с учетом каскадных процессов теперь будет рассчитываться по формуле

$$
\eta_{\mathrm{FP}}=N_{C} \sigma_{d} v
$$

Поскольку, как рассчитано выше для электронов с энергией $3.5 \mathrm{MэB}, \sigma_{d}=18$ барн и $v=2$, темп генерации
ПФ из (6) будет составлять $2.0 \mathrm{~cm}^{-1}$ (см. таблицу). Итак, расчетное увеличение параметра $\eta_{\text {FP при увели- }}$ чении энергии электронов от 0.9 до $3.5 \mathrm{MэВ} \mathrm{составляет}$ $\sim 2$ раза. Напомним, что экспериментальное увеличение параметра $\eta_{e}$ составляет 4 раза. Таким образом, простым увеличением количества дефектов (скорости генерации радиационных дефектов даже с учетом каскадных процессов) экспериментальные данные объяснить довольно сложно. Попробуем рассмотреть „качество“ образующихся РД, а именно среднее расстояние между компонентами образующихся ПФ. При увеличении энергии бомбардирующих электронов от 1 до $3.5 \mathrm{MэB} \mathrm{в} \mathrm{спектре}$ ПВА изменяется только участок, соответствующий почти лобовым столкновениям. Этот участок „высокоэнергетичных“ („горячих“) ПВА простирается примерно от 300 до 1000 эВ. С ростом энергии ПВА увеличивается среднее расстояние между генетически родственными компонентами ПФ. Для расчета энергетических зависимостей пробегов ионов обычно используется пакет компьютерного моделирования, называемый SRIM [20], основанный на теоретических моделях, описанных в работе [19]. На рис. 4 представлена зависимость пробега ионов ПВА углерода и кремния в карбиде кремния от энергии ПВА для диапазона 50-1000 эВ, рассчитанная по программе SRIM. Известно, что эффективность вторичного дефектообразования определяет главным образом, насколько полно происходит диссоциация (разделение) первично созданных генетически родственных пар Френкеля: вакансия (V) - межузельный атом (I). В ходе диссоциации возможно изменение знака заряда компонент пары. Последнее определяет характер взаимодействия V и I, а также позволяет определить некий радиус рекомбинации ПФ $(r)$ (расстояние между компонентами ПФ, критичное для их рекомбинации). В этой связи решающими оказываются два обстоятельства: распределение первично созданных ПФ по расстоянию между компонентами и наличие электронов и дырок, обусловливающих перезарядку. Заметим, что в карбиде кремния энергия образования одной электронно-дырочной пары (ЭДП) составляет 9.6 эВ; поэтому темп генерации ЭДП в $\mathrm{SiC}$ при электронном облучении на наших уско-

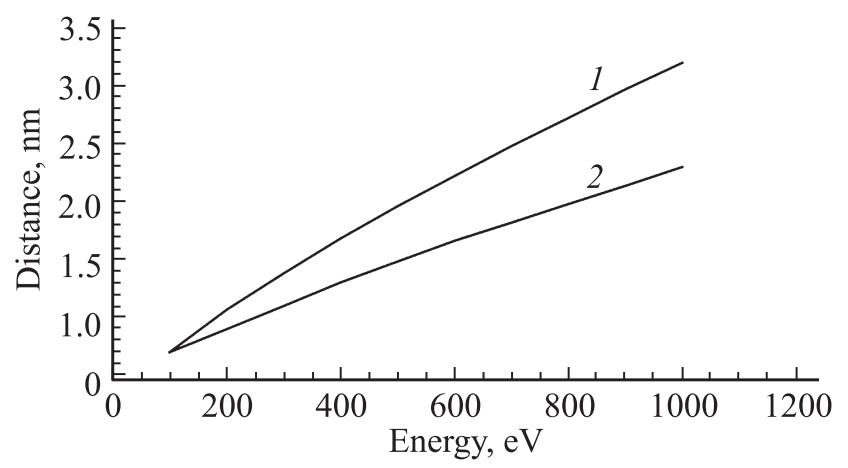

Рис. 4. Зависимость пробега ПВА углерода (1) и кремния (2) в карбиде кремния от энергии ПВА, рассчитанная по программe SRIM [19]. 
рителях оценивается величиной $\sim 10^{20} \mathrm{~cm}^{-3} \cdot \mathrm{c}^{-1}[3,6]$. Для карбида кремния нам не удалось найти теоретических работ по зависимости радиуса рекомбинации от зарядового состояния компонент. Для кремния же эти работы хорошо известны. Так, в работах [21-23] указывается, что $\mathrm{V}$ и I в момент рождения нейтральны, и радиус рекомбинации пары $\left[\mathrm{V}^{0} \mathrm{I}^{0}\right]$ составляет $\sim 2 a$, где $a-$ постоянная решетки (для кремния $a_{\mathrm{Si}} \sim 0.543 \mathrm{нм}$ ). При захвате вакансией электрона пара $\left[\mathrm{V}^{-} \mathrm{I}^{0}\right]$ диссоциирует при $r>4 a$. При полной перезарядке пары $\left[\mathrm{V}^{-} \mathrm{I}^{+}\right]$расстояние возрастает, и $r>7 a$. Попробуем оценить радиус рекомбинации дефектов в подрешетке углерода исходя из степени диссоциации этих дефектов. Большинство „горячих“ ПВА будет удаляться от места образования, и пара Френкеля разделится на свободную вакансию и межузельный атом. Как следствие с ростом энергии атомов отдачи увеличивается доля диссоциирующих ПФ $f_{\text {FP. }}$

$$
f_{\mathrm{FP}}=\eta_{e} / \eta_{\mathrm{FP}} .
$$

Скорость удаления доноров в $n$-SiC на начальных стадиях облучения может служить мерой образования в подрешетке углерода этих „далеких“ пар, т.е. разделившихся на изолированные вакансии и межузельные атомы. При облучении электронами с энергией 0.9 МэВ скорость удаления доноров составляет $0.1 \mathrm{~cm}^{-1}$ (рис. 2), а темп генерации ПФ в подрешетке углерода $1.0 \mathrm{~cm}^{-1}$, поэтому доля разделившихся (диссоциировавших) ПФ составляет $10 \%$. Оценим характерную энергию ПВА углерода, при которой в правой высокоэнергетической части спектра оказывается 10\% ПВА. Учтем, что энергетический спектр ПВА или количество атомов, первично выбитых релятивистским электроном из своих равновесных положений, распределяется приблизительно по закону обратного квадрата энергии. При такой зависимости легко оценить долю ПВА в правой части спектра (долю разделившихся ПФ):

$$
f_{\mathrm{FP}}=\left(1 / E_{\mathrm{ch}}-1 / E_{2}\right) /\left(1 / E_{1}-1 / E_{2}\right),
$$

где $E_{1}=18$ эВ (пороговое значение дефектообразования); $E_{2}=307$ эВ - максимальное значение энергии ПВА, оцененное по формуле (2) для энергии бомбардирующих электронов, равной 0.9 МэВ. При этих условиях величина характерной энергии $E_{\mathrm{ch}}$ составляет 122 эВ. Атомы отдачи углерода, получившие энергию 122 эВ и выше, будут покидать зону спонтанной рекомбинации, обеспечивая формирование стабильных РД, приводящих к компенсации проводимости $n$ - SiC. Используя результаты расчетов по программе SRIM (рис. 4), легко оценить пробег ионов углерода с энергией 122 эВ в $\mathrm{SiC}$. Этой энергии соответствует пробег, равный $\sim 8 \AA$, который можно приписать и радиусу рекомбинации. Поскольку расстояние между ближайшими атомами углерода в $4 H$-SiC составляет $a_{0} \sim 1.85 \AA[24,25]$, радиус рекомбинации составляет примерно величину, равную четырем $a_{0}$. Если пользоваться аналогией с кремнием $[21,22]$, о которой говорилось выше, то четырех- кратное превышение радиуса рекомбинации по отношению к постоянной решетки может свидетельствовать о рекомбинации генетически родственных компонент пар Френкеля в подрешетке углерода в состоянии, при котором вакансия углерода захватила электрон $\left[\mathrm{V}^{-} \mathrm{I}^{0}\right]$. C ростом энергии бомбардирующих электронов, увеличивается доля ПВА, получивших энергию, большую чем 122 эВ. Так, для энергии $3.5 \mathrm{MэВ} \mathrm{доля} \mathrm{разделив-}$ шихся (диссоциировавших) ПФ, согласно формуле (8), составляет порядка $15 \%$. При этом отношение скорости удаления носителей заряда, полученное экспериментально, к скорости генерации пар Френкеля, рассчитанное с учетом коэффициента размножения, составляет, согласно формуле (7), порядка 15\%. Таким образом, учет „количества“ образующихся дефектов - ПФ (с учетом каскадных процессов) и степени их диссоциации на изолированные компоненты в зависимости от энергии ПВА позволил непротиворечиво объяснить наблюдаемую экспериментальную зависимость скорости удаления доноров в $n-4 H-\mathrm{SiC}(\mathrm{CVD})$ от энергии бомбардирующих электронов.

\section{5. Заключение}

Проведенное исследование показало наличие сильной зависимости компенсации проводимости $n-4 H-\mathrm{SiC}$ (CVD) от энергии бомбардирующих электронов (в диапазоне 0.9-3.5 МэВ). При этом сечение образования первичных радиационных дефектов (пар Френкеля в подрешетке углерода), ответственных за компенсацию, в этом диапазоне практически не зависит от энергии. Показано, что основным фактором является энергия первично выбитых атомов ПВА. С повышением энергии ПВА, во-первых, начинают развиваться каскадные процессы, которые приводят к увеличению количества ПВА, во-вторых, увеличивается среднее расстояние между генетически родственными ПФ и как следствие увеличивается доля нерекомбинировавших при облучении ПФ. Оценено значение радиуса рекомбинации ПФ в подрешетке углерода $(\sim 0.8$ нм$)$, которое позволяет косвенно уточнить зарядовое состояние рекомбинирующих компонент. Прямое определение концентраций радиационных дефектов в подрешетке углерода в $n-\mathrm{SiC}(\mathrm{CVD})$ при модельном облучении $\left(E_{e}=0.9 \mathrm{MэB}\right)$ и при „надпороговом“ (по отношению к образованию каскада) облучении с коэффициентом размножения порядка $2\left(E_{e}=3.5 \mathrm{MэВ)} \mathrm{требует} \mathrm{дополнительных} \mathrm{иссле-}\right.$ дований.

Настоящая работа выполнена при поддержке гранта РНФ (проект № 16-12-10106 „Радиационная стойкость карбида кремния и приборы на его основе для экстремальной электроники“). 


\section{Список литературы}

[1] J. Lutz, H. Schlangenotto, U. Scheuermann, Rik De Doncker. Semiconductor Power Devices Physics. Characteristics, Reliability (Springer Verlag, Berlin Heidelberg, 2011).

[2] В.А. Козлов, В.В. Козловский. ФТП, 35, 769 (2001).

[3] L.F. Zakharenkov, V.V.Kozlovski, B.A. Shustrov. Phys. Status Solidi A, 117, 85 (1990).

[4] C. Claeys, E. Simoen. Radiation Effects in advanced semiconductor materials and devices (Springer Verlag, Berlin Heidelberg, 2002).

[5] A. Holmes-Siedle, L. Adams. Handbook of radiation effects (Oxford, Oxford University, 1993).

[6] V. Kozlovski, V. Abrosimova. Radiation Defect Engineering (World Scientific, 2005).

[7] W.J. Choyke. Inst. Phys. Conf. Ser., 31, 58 (1977).

[8] V.V. Kozlovski, N.B. Strokan, A.M. Ivanov, A.A. Lebedev, V.V. Emtsev, G.A. Oganesyan, D.S. Poloskin. Physica B, 404, 4752 (2009).

[9] A. Castaldini, A. Cavallini, L. Rigutti, F. Nava, S. Ferrero, F. Giorgis. J. Appl. Phys., 98, 053706 (2005).

[10] В.В. Козловский, В.В. Емцев, К.В. Емцев, Н.Б. Строкан, А.М. Иванов, В.Н. Ломасов, Г.А. Оганесян, А.А. Лебедев. ФТП, 42, 243 (2008).

[11] J.W. Steeds, F. Carosella, G.A. Evans, M.M. Ismail, L.R. Danks, W. Voegeli. Mater. Sci. Forum, 353-356, 381 (2001).

[12] J.W. Steeds, G.A. Evans, S. Furkert, M.M. Ismail, L.R. Danks, W. Voegeli, F. Carosella. Diamond Relat. Mater., 11, 1923 (2002).

[13] H. Kaneko, T. Kimoto. Appl. Phys. Lett., 98, 262106 (2011).

[14] V.V. Kozlovski, A.A. Lebedev, E.V. Bogdanova. J. Appl. Phys., 117, 155702 (2015).

[15] http://cree.com/

[16] W.A. MacKinley, H. Feshbach. Phys. Rev., 74, 1759 (1948).

[17] G.J. Dienes, G.H. Vineyard. Radiation Effects in Solids (Interscience Publishers, N. Y., 1957).

[18] G.H. Kinchin, R.S. Pease. Rep. Prog. Phys., 18, 1 (1955).

[19] J.F. Ziegler, J.P. Biersack, U. Littmark. The Stopping and Range of Ions in Matter (Pergamon, N.Y., 1985).

[20] http://www.srim.org

[21] А.М. Иванов, И.Н. Ильяшенко, Н.Б. Строкан, Б. Шмидт. ФТП, 29, 543 (1995).

[22] В.В. Емцев, Т.В. Машовец, В.В. Михнович. ФТП, 26, 22 (1992).

[23] A. Nylandsted Larsen, A. Mesli. Electron and Proton Irradiation of Silicon. Chap. 2. In: Defects in Semiconductors, ed. by L. Romano, V. Privitera and Ch. Jagadish [Ser. Semiconductors and Semimetals, vol. 91] (Elsevier, 2015) p. 47.

[24] M.E. Levinshtein, S.L. Rumyantsev, M.S. Shur. Properties of Advanced Semiconductor Materials: GaN, AlN,InN, BN, SiC, SiGe (John Wiley \& Sons, Inc., 2001).

[25] N. Iwamoto, B.G. Svensson. Point Defects in Silicon Carbide. Chap. 10. In: Defects in Semiconductors, ed. by L. Romano, V. Privitera and Ch. Jagadish [Ser. Semiconductors and Semimetals, vol. 91] (Elsevier, 2015) p. 369.

\section{Effect of the bombarding electrons energy on epitaxial layer $n-4 H$-SiC (CVD) conductivity}

V.V. Kozlovski' , A.A. Lebedev' ${ }^{2}$, A.M. Strel'chuk ${ }^{2}$, K.S. Davidovskaya ${ }^{2}$, A.E. Vasil'ev' ${ }^{1}$, L.F. Makarenko ${ }^{3}$

${ }^{1}$ Peter the Great St. Petersburg

Polytechnic University,

195251 St. Petersburg, Russia

${ }^{2}$ Ioffe Institute,

194021 St. Petersburg, Russia

${ }^{3}$ Belorussian State University,

220030 Minsk, Belarus

Abstract The study of the electrical characteristics of epitaxial layers of $n-4 H-\mathrm{SiC}$ (CVD) irradiated by electrons with energies of 0.9 and $3.5 \mathrm{MeV}$ were made. It is shown that the removal rate of the donor increases almost 4 times with the incident electron energy increases by 4 times. Although the cross section of primary radiation defects (Frenkel pairs in carbon sublattice) arising, responsible for the compensation of material conductivity in this range is practically independent of energy. It is assumed that the cause of the observed differences is the influence of the primary knocked atoms. Firstly, with increasing energy PVA starting to affect the cascade processes. Secondly, increasing the average distance between genetically related Frenkel pairs and as a consequence, the share does not recombine during irradiation defects. It was estimated Frenkel pair recombination radius in carbon sublattice and possible charge state of the recombining component. 